\title{
Depression as a Risk Factor for Cognitive Impairment in Later Life: The Health In Men Cohort Study
}

\author{
Osvaldo P. Almeida, $\mathrm{PhD}^{1,2,3}$ \\ Graeme J. Hankey, MD ${ }^{4,5}$ \\ Bu B. Yeap, $\mathrm{PhD}^{4,6}$ \\ Jonathan Golledge, MChir ${ }^{7,8}$ \\ Leon Flicker, $\mathrm{PhD}^{2,4,10}$
}

${ }^{1}$ School of Psychiatry \& Clinical Neurosciences, University of Western Australia, Perth, Australia

${ }^{2}$ WA Centre for Health \& Ageing, Centre for Medical Research, Perth, Australia

${ }^{3}$ Department of Psychiatry, Royal Perth Hospital, Perth, Australia

${ }^{4}$ School of Medicine and Pharmacology, University of Western Australia, Perth, Australia

${ }^{5}$ Department of Neurology, Sir Charles Gairdner Hospital, Perth, Australia

${ }^{6}$ Department of Endocrinology, Fremantle Hospital, Fremantle, Australia

${ }^{7}$ Queensland Research Centre for Peripheral Vascular Disease, College of Medicine and Dentistry, James Cook University, Townsville, Australia

${ }^{8}$ Department of Vascular and Endovascular Surgery, The Townsville Hospital, Townsville, Australia

${ }^{9}$ Department of Geriatric Medicine, Royal Perth Hospital, Perth, Australia

Running title: Depression and Cognitive Impairment

Keywords: Depression, cognitive impairment, dementia, epidemiology, risk factors.

Funding: This work was funded through competitive project grants from the National Health and Medical Research Council of Australia (NHMRC), numbers 279408, 379600, 403963, 513823, 540403, 540504, 540405, 634492, 1021416, 1045710 and 1060557. The sponsors had no role in the design and conduct of the study; collection, management, analysis, and interpretation of the data; or preparation, review, or approval of the manuscript.

Key points:

- Clinically significant symptoms of depression affect 5.5\% of older men, with another $11.5 \%$ having experienced depression in the past.

- History of past depression does not increase the risk of mild cognitive impairment or of major cognitive impairment over a follow up period of 5 years.

- History of current depression more than doubles the risk of major cognitive impairment over 5 years, which suggests that depression in later life may represent a prodromal phase of cognitive impairment.

Word count: 3454

Correspondence

Prof. Osvaldo P. Almeida, School of Psychiatry \& Clinical Neurosciences (M573), University of Western Australia, 35 Stirling Highway, Crawley, Perth, WA 6009, Australia. Email: osvaldo.almeida@uwa.edu.au. 


\section{ABSTRACT}

Background: Depression is an established risk factor for dementia in later life, but it is unclear if this relationship is causal. This study aimed to determine if clinically significant depressive symptoms are likely to be causally related to cognitive impairment in later life.

Methods: Observational cohort study of 4568 men aged 70-89 years living Perth, Western Australia, who were free of cognitive impairment at the beginning of follow up. Current clinically significant depressive symptoms were defined by a score of 7 or more on the Geriatric Depression Scale 15 items. Past depression was ascertained via electronic medical records, by self-report, or use of antidepressants. A score of 27 or less on the Telephone Interview for Cognitive Status modified (TICS) or a recorded diagnosis of dementia in electronic medical records established the presence of cognitive impairment.

Results: During the 5-year follow up, 534 men developed cognitive impairment, 811 died and 1455 were lost. The presence of clinically significant depressive symptoms at study entry was associated with increased risk rate $(R R)$ of cognitive impairment $(R R=2.59,95 \%$ confidence interval: 95\%CI=1.57-4.27), death $(\mathrm{RR}=5.07,95 \% \mathrm{CI}=3.32-7.75)$ and loss to follow up $(\mathrm{RR}=2.03$, 95\%CI=1.32-3.13). These associations remained statistically significant after adjustment for age, country of birth, education, smoking history, and prevalence hypertension, diabetes, coronary heart disease and stroke. History of past clinically significant depressive symptoms was not associated with incident cognitive impairment $(\mathrm{RR}=1.09,95 \% \mathrm{CI}=0.78-1.52)$.

$\underline{\text { Conclusions: }}$ The lack of association between past depression and cognitive impairment suggests that the link between depression and cognitive impairment is not causal and that the presence of clinically significant depressive symptoms in later life may herald the onset of cognitive impairment in at least some people. 


\section{INTRODUCTION}

Dementia is a leading cause of years of life lost due to disability and a major public health challenge for the twenty-first century (Murray et al., 2012, Sloane et al., 2002). Among the strategies to reduce the global burden of dementia, prevention currently has greater potential than cure, and certainly greater value than current symptomatic treatments. Potentially modifiable risk factors for Alzheimer's disease (AD), the most frequent cause of dementia, include diabetes mellitus, midlife hypertension or obesity, physical inactivity, smoking, limited education and depression (Norton et al., 2014). If depression is causally linked to AD, it may account for $8 \%$ of all cases of $\mathrm{AD}$ worldwide, indicating that nearly 1 in every 10 cases of $\mathrm{AD}$ could be averted or deferred if depression were successfully treated or prevented (Norton et al., 2014).

Although there is no proof, by means of randomized controlled trials, that preventing or treating depression reduces the incidence of dementia, a systematic review and meta-analysis of 23 cohort studies reported that depression after age 50 years was associated with an increased odds of allcause dementia [odds ratio $(\mathrm{OR})$ and 95\% confidence interval $(95 \% \mathrm{CI})$, OR=1.85, 95\%CI=1.672.04)], $\mathrm{AD}(\mathrm{OR}=1.65,95 \% \mathrm{CI}=1.42-1.92)$ and vascular dementia (VD) $(\mathrm{OR}=2.52,95 \% \mathrm{CI}=1.77-$ 3.59) (Diniz et al., 2013). Whilst these results are consistent with the hypothesis that depression causes dementia, the diagnosis of depression in most studies relied on the use of depression scales rather than validated diagnostic criteria, confounding could potentially explain the findings (i.e., common risk factors associated with both depression and dementia), and the time-gap between exposure to depression and onset of dementia varied widely (1 to 17 years). Hence, depressive symptoms could simply herald the early stages of an ongoing dementia process rather than being the cause of it. Indeed, only 2 of the 23 studies included in the meta-analysis provided follow up data for periods longer than 9 years (Diniz et al., 2013). 
We aimed to circumvent this uncertainty by investigating if both early and late-life depression are associated with the development of cognitive impairment in a community representative sample of older Western Australian men (Norman et al., 2009). We hypothesized that both past and current depression would increase the risk of incident cognitive impairment amongst participants.

\section{METHODS}

\section{$\underline{\text { Study design and setting }}$}

This is a cohort study of a community-derived sample of older men living in the Perth metropolitan region of Western Australia.

\section{$\underline{\text { Participants }}$}

The sample consisted of 4568 men aged 70-89 years who completed the second wave of assessments of the Health In Men Study (HIMS) in 2001-2004. Briefly, HIMS is an ongoing cohort study of a random community sample of 12,203 older men recruited between 1996-1998 for a study of abdominal aortic aneurysm. An additional third wave of clinical assessments took place in 2008. Details about the study design and cohort have been described elsewhere (Almeida et al., 2014, Norman et al., 2009). Eligible men had Mini-Mental State Examination (MMSE) scores $\geq 24$ (Crum et al., 1993) at the 2001-2004 assessment and no prior recorded history of dementia in the Western Australian Data Linkage System (WADLS) before the 2001-2004 assessment. WADLS contains health morbidity data for all hospital contacts in Western Australia going back to 1980, as well as all mental health contacts with both inpatient and public outpatient services since 1966. The Australian Bureau of Statistics provides the mortality data linked to WADLS (http://www.abs.gov.au/ausstats/abs@.nsf/mf/3303.0) (Holman et al., 2008, Holman et al., 1999). The recorded diagnoses follow the International Classification of Diseases (ICD) coding system of the $9^{\text {th }}$ edition until $30 / 06 / 2008$ and $10^{\text {th }}$ edition thereafter. The following codes indicated the presence of dementia: 290.x, 294.1 and 294.2 (ICD-9), and F00, F01, F02 and F03 (ICD-10). 
This study followed the principles of the Declaration of Helsinki and was approved by the Ethics Committees of the University of Western Australia and of the Department of Health of Western Australia. Participants provided written informed consent to participate.

\section{$\underline{\text { Healthy participant bias }}$}

Men who consented to join HIMS were healthier than other eligible men living in the community (Almeida et al., 2014), and those who completed the second assessment had less comorbidities than their surviving counterparts lost throughout the follow up period (Almeida et al., 2014, Almeida et al., 2008a).

\section{$\underline{\text { Explanatory variables }}$}

The principal explanatory variable of the study was the presence of past or current depression at the start of the follow up period. We used three complementary strategies to identify men with past depression: (i) recorded diagnosis of a depressive episode in WADLS before the date of assessment (ICD-9 codes 296.2, 296.3, 296.82, 296.90, 298.0 and 311, and ICD-10 codes F32, F33, F34.1 and F38.10), (ii) response in the affirmative to the question 'Have you ever had treatment for an emotional or nervous illness such as depression?', or (iii) use of an antidepressant at the time of assessment. We judged that participants showed evidence of current clinically significant symptoms of depression if they scored 7 or more on the 15-item version of the Geriatric Depression Scale (GDS-15) at the time of assessment (Almeida and Almeida, 1999). All other men were considered to have not experienced a clinically significant episode of depression.

At the start of the follow up period participants completed a health questionnaire that collected information about their age (in years), place of birth (Australia or other), educational attainment (incomplete vs complete high school education), and smoking (never, past or current). In addition, 
we considered that participants had hypertension if their systolic blood pressure was $\geq 140 \mathrm{mmHg}$ or their diastolic blood pressure was $\geq 90 \mathrm{mmHg}$, or if they reported having been advised by their doctor that they had hypertension. The presence of diabetes was ascertained by asking participants if a doctor had ever told them that they had diabetes or if they reported treatment to lower blood sugar. Similarly, men indicated whether a doctor had ever told them that they had had a stroke, or a heart attack or angina (which we considered indicative of the presence of coronary heart disease).

\section{Follow-up}

We invited participants to complete an additional assessment with the Telephone Interview for Cognitive Status modified (TICS) during 2008 and used this information to assign men to three groups: normal cognitive function (TICS > 31), mild cognitive impairment (MCI; $27<$ TICS $\leq 31$ ) and cognitive impairment (TICS $\leq$ 27) (Knopman et al., 2010). These cut-points have been associated with sensitivity and specificity of $71 \%$ and $78 \%$ to separate MCI from normal cognitive function, and 69\% and 71\% to separate MCI from dementia (Knopman et al., 2010). In addition, we

identified participants who did not return for assessment but received a WADLS diagnosis of dementia between the 2001-4 and the 31st December 2008. These men were assigned to the 'cognitive impairment' group. Finally, we used the WADLS to identify men who died between the date of enrolment and the 31st December 2008.

\section{$\underline{\text { Outcomes }}$}

The primary outcome was incident cognitive impairment and mild cognitive impairment by the 31st December 2008, as measured by the TICS during 2008 or a WADLS diagnosis of dementia between the 2001-4 and the 31st December 2008.

$\underline{\text { Statistical methods }}$ 
Data were managed and analysed with the statistical package Stata/IC 13.1 (StataCorp LP, 2014). We used descriptive statistics to summarise categorical data as count and proportions (\%), and continuous variables as mean, range, and standard deviation of the mean (SD). We used the Pearson chi-square statistic $\left(X^{2}\right)$ to determine the probability that the distribution of exposures among people with past, current and no history of depression could be attributed to chance, and reported the number of degrees of freedom (df) and the probability (p-value) of the associations having arisen as a result of chance. We employed multinomial logistic regression to calculate the risk rate ratio (RR) and respective 95\% confidence interval (95\%CI) of MCI, cognitive impairment, death and unavailability for the follow up cognitive assessment in 2008 according to depression history and other explanatory variables. The RR of the clinical outcomes was later adjusted for other measured factors. Alpha was set at 0.05 and all tests reported are two-tailed.

\section{RESULTS}

The mean \pm SD age of the 4568 men who took part in this study was $77.1 \pm 3.6$ years. Of these, 253 (5.5\%) had clinically significant depressive symptoms at the time of assessment, with another 527 (11.5\%) showing evidence of past depression.

Table 1 summarises the baseline sociodemographic and clinical characteristics of participants according to their history of depression. Men with clinical significant depressive symptoms at baseline were older and more likely to be born outside Australia, poorly educated, smokers, diabetic with a history of coronary heart disease or stroke. In addition, of the 527 men assigned to the 'past depression’ group, 227 (43.1\%) were using an antidepressant. Forty-one (16.2\%) of the 253 participants with GDS-15 $\geq 7$ were also using an antidepressant.

TABLE 1 
Table 2 displays the distribution of baseline sociodemographic and clinical characteristics of participants according to the cognitive and survival outcomes of men by the $31^{\text {st }}$ December 2008 (mean \pm SD follow up time $=5.2 \pm 0.8$ years). The risk of MCI, cognitive impairment, death and unavailability for the follow up assessment increased with increasing age and was particularly high for those older than 80 years. The RR of non-availability for follow up was higher among participants not born in Australia $(\mathrm{OR}=1.25$; 95\%CI=1.06-1.45), whereas the odds ratio of $\mathrm{MCI}$, cognitive impairment, death and unavailability was lower for men with complete compared with incomplete high school education. Diabetes increased the RR of cognitive impairment, death and unavailability, but not of MCI. The RR of death also increased with history of current smoking, diabetes, coronary heart disease and stroke. Similarly, unavailability was more frequent among current smokers and men with clinical history of diabetes and stroke (Table 2).

\section{TABLE 2}

History of past depression did not increase the RR of MCI or cognitive impairment, but current depression did increase the $\mathrm{RR}$ of future cognitive impairment $(\mathrm{RR}=2.59,95 \% \mathrm{CI}=1.57-4.27$; $\mathrm{RR}=2.07,95 \% \mathrm{CI}=1.24-3.45$ after adjustment for other measured factors). Past and current depression at the beginning of follow up were associated with increased risk of death, whereas current depression was also associated with unavailability at the follow up assessment (Table 3). We repeated these analyses after the exclusion of men who were using an antidepressant at the time of entry into the study: the results remained largely unchanged (Table 3).

Post hoc analyses showed that there was no obvious 'dose effect' of depression severity on the future development of cognitive impairment: compared with participants who scored $<5$ on the GDS-15 (i.e., no depression), the crude RR of cognitive impairment was 2.11 (95\%CI=1.40-3.18) for men with GDS-15 scores between 5 and 9 (mild to moderate depression) and 2.70 
(95\%CI=1.16-6.28) for those with GDS-15 $\geq 10$ (severe depression). After statistical adjustment for age group, country of birth, education, smoking history, and prevalence hypertension, diabetes, coronary heart disease and stroke, the respective RR of cognitive impairment were 1.83 (95\%CI=1.21-2.79) and $2.24(95 \% \mathrm{CI}=0.95-5.31)$.

\section{TABLE 3}

\section{DISCUSSION}

The results of this study showed that history of past depression was not associated with the risk of incident cognitive impairment (either mild or major cognitive impairment) in older men. In contrast, evidence of clinically significant symptoms of depression at the start of the follow up period more than doubled the risk of cognitive impairment over the subsequent 5 years, although it had no effect on the risk of mild cognitive impairment. These findings were independent of other measured factors, as well as of death and loss to follow up. As MCI represents an intermediate evolutionary

state between normality and overt cognitive impairment (Petersen et al., 2014), the lack of association between current depression and future MCI, as well as the lack of association between past depression and cognitive impairment, suggests (but does not prove) that the link between depression and cognitive impairment is not causal.

\section{$\underline{\text { Strengths and limitations }}$}

Before discussing the implications of our findings, we will consider the limitations of our study design and how these, chiefly potential for bias and confounding, may have influenced the results.

Participants were derived from a large community-representative sample of older men for whom a wealth of questionnaire and health service use data was available (Norman et al., 2009, Prina et al., 2013). Men who completed the 2001-2004 wave of assessments for HIMS were healthier than those 
who were still alive but were not available (Almeida et al., 2014, Almeida et al., 2008b). The implication of such healthy participant bias is that fewer men with depression would have joined this survey, leading to a loss of power to detect future associations with depression (type II error).

We are confident that by using the MMSE and WADLS we excluded from the analyses prevalent cases of cognitive impairment, but we are less certain that all past depressive episodes would have been identified correctly by means of self-report and WADLS. As WADLS only retrieves data from public outpatient and hospital services, less severe cases of depression managed in the private sector or at primary care level would not have been recorded (Holman et al., 2008, Holman et al., 1999). Although self-report would have contributed to mitigate this type of bias, the accuracy and completeness of the approach we used to ascertain past depression is yet to be tested. In addition, the sensitivity of WADLS in identifying people with dementia is low (Zilkens et al., 2009), so that potential cases of cognitive impairment may have gone unidentified amongst those who died or were not available for the cognitive assessment. If that were the case, then the number of people with cognitive impairment could have been larger, and this would have increased the power of the study to identify associations with depression. Notwithstanding such a caveat, our study had sufficient power to declare as significant risk rates of the order of 1.5 , which suggests that this potential misclassification of cases of cognitive impairment would have had limited bearing on the clinical significance of the findings.

Despite prospective follow-up of all participants, 30\% of them did not complete the cognitive assessment and 18\% died during the 5-year follow up. If men with history of past depression who are at greater risk of developing cognitive impairment died early, then survivorship bias could potentially account for the lack of association between past depression and cognitive impairment. Although we are unable to dismiss this possibility, this would seem unlikely to explain our results, as the effect size of the observed association between past depression and cognitive impairment was 
negligible (i.e., nearly all cases who died would have had to have experienced past depression and developed cognitive impairment for the effect to become statistically significant), and the effect was not modified by the exclusion from the sample of men using antidepressants. Nonetheless, we concede that if a large proportion of men with past depression were misclassified as non-cases (i.e., false negatives), this would have led to a loss of power to investigate the association with future cognitive impairment.

It may also seem surprising that stroke was not associated with cognitive impairment at follow up. Severe and more disabling strokes are commonly associated with significant disability and mortality (which is consistent with the increased risk of death and unavailability in our sample), so that survivors are often at the more benign end of disease spectrum. Moreover, our study was not powered to investigate the association between stroke and cognitive impairment, as most men (60\%) with past history of stroke either died or were unavailable for follow up.

If, in at least some instances, depression is a prodromal expression of dementia in older age, then a follow up period of 5 years should have been sufficiently long for the clinical manifestations of dementia to become apparent (Amieva et al., 2008). In contrast, if depression is a true risk factor for dementia, then more prolonged follow up could have yielded more cases. Our study collected information about past depression (i.e., more than 5 years before the onset of cognitive impairment) and found no evidence of such a link. We acknowledge, however, that we did not have access to information about the age of onset of depression, the number of past episodes, or the type and duration of antidepressant treatments used. Hence, we were unable to determine if any of these factors could have potentially modified the association between depression and incident cognitive impairment. Furthermore, we acknowledge that, like other surveys, our definition of 'current depression' relied on the use of a depression rating scale rather than a structured clinical interview leading to a diagnosis of major depression according to accepted criteria. 
There is also the potential issue of confounding associated with unmeasured factors, such as socioeconomic status, physical activity, and access to health services. It is conceivable that such factors could explain some of the association between current depression and future cognitive impairment (Lautenschlager et al., 2008), although it seems improbable that they could explain all of it, particularly because our statistical adjustments for other measured factors did not have a pronounced effect on the results.

Finally, we acknowledge that the use of multinomial logistic regression does not take into account time to event, which could not be accurately assessed in this survey, and that, as our data are restricted to older men, we cannot be certain that the same findings would be observed in women.

\section{Interpretation of the results}

We designed this study to determine if depression is causally related to the development of cognitive impairment in later life. Taken together, our results seem to have answered this question in the negative. Past studies have identified depression as a risk factor for cognitive impairment, although most have been limited to late-life depression (Diniz et al., 2013). Li and colleagues examined data from 3410 adults aged 65 years who were followed for 7 years as part of the Adults Changes in Thought Study (Li et al., 2011). They used the Center for Epidemiologic Studies Depression Scale (CES-D-11) to assess depressive symptoms and considered a score of 11 or greater indicative of clinically significant symptoms. Participants self-reported history of past depression and the diagnosis of dementia followed clinical assessment and DSM-IV criteria. The hazard ratio (HR) of dementia associated with baseline depression was 1.7 (95\%CI=1.4-2.1), although clinically significant depression with onset before age 50 years did not increase dementia hazard $(\mathrm{HR}=1.1,95 \% \mathrm{CI}=0.8-1.5)$. The authors concluded that some cases of late life depression represent a prodromal manifestation of dementia and that depression per se does not increase 
dementia risk (Li et al., 2011). Similar findings have since been reported by others (Vilalta-Franch et al., 2013), and are consistent with our own. Compared with the Adults Changes in Thought Study, HIMS has the merit of having had access to the Western Australia Data Linkage System to optimise the identification of cases of past depression as well as of dementia.

We also investigated the effect of severity of symptoms in order to clarify if the association between prevalent depression and incident cognitive impairment was subject to a dose-effect that could be supportive of a causal link: we found no compelling evidence of such a link. Others have reported some evidence for a dose-effect with every 10-points increase in CES-D scores (Saczynski et al., 2010), although the HR of dementia reported was averaged (HR=1.5, 95\%CI=1.2-1.8) and the reference group had no depression (i.e., CES-D $\leq 10$ ). In other words, these results may have simply reflected the average effect of having depression compared with no depression.

It is conceivable that our results could reflect the fact that depression with onset in early and late life have distinct pathogeneses. The 'vascular depression' hypothesis implies that depression with late onset is commonly due to cerebrovascular disease (Alexopoulos et al., 1997), which could diminish brain reserve and bring forward the onset of dementia (Snowdon et al., 1997). Empirical evidence in support of the vascular hypothesis of late life depression is not compelling (Almeida, 2008), but it highlights the fact that some older adults who display clinically significant symptoms of depression in later life carry greater disease burden in their brains than those who do not (Kohler et al., 2010). In this case, depressive symptoms (not necessarily a major depressive episode) would represent a clinical manifestation of the underlying brain abnormalities that also lead to cognitive impairment (Korten et al., 2014).

In summary, the results of our study are consistent with the hypothesis that depression in later life is a prodromal manifestation of cognitive impairment rather than the cause of it. If future studies 
prove this to be true, the successful treatment or prevention of depression in early life would not be expected to affect the prevalence of cognitive impairment in later life. Instead, depression in older age might identify a group of people who may benefit from interventions designed to delay progression to cognitive impairment over time, in the same way as MCI. 


\section{Original publication}

The authors declare this report contains original unpublished work that is not being considered for publication elsewhere.

\section{Declaration of interests}

The authors declare they have no conflicts of interest.

\section{$\underline{\text { Roles and responsibilities }}$}

OPA conceived and designed the study. OPA, GJH, BBY, JG and LF performed the experiments. OPA analysed the data and drafted the manuscript. OPA, GJH, BBY, JG and LF reviewed the manuscript critically and approved its submission to the Journal.

\section{Ethics approval}

The Human Research Ethics Committees of the Royal Perth Hospital and of the Department of Health of Western Australia approved the research protocol and procedures of the study, which follow the principles of the Declaration of Helsinki. All participants provided written informed consent.

\section{$\underline{\text { Access to data }}$}

Consent procedures and ethics approval restrict access to the data to named investigators.

\section{$\underline{\text { Provenance and peer review }}$}

This is an investigator-initiated study; not commissioned. 


\section{REFERENCES}

Alexopoulos GS, Meyers BS, Young RC, et al. 1997. 'Vascular depression' hypothesis. Arch Gen Psychiatry 54: 915-922.

Almeida OP. 2008. Vascular depression: myth or reality? Int Psychogeriatr 20: 645-652.

Almeida OP, Almeida SA. 1999. Short versions of the geriatric depression scale: a study of their validity for the diagnosis of a major depressive episode according to ICD-10 and DSM-IV. Int J Geriatr Psychiatry 14: 858-865.

Almeida OP, Hankey GJ, Yeap BB, et al. 2014. Mortality among People with Severe Mental Disorders Who Reach Old Age: A Longitudinal Study of a Community-Representative Sample of 37892 Men. PLoS One 9: e111882.

Almeida OP, Mccaul K, Hankey GJ, et al. 2008a. Homocysteine and depression in later life. Arch Gen Psychiatry 65: 1286-1294.

Almeida OP, Yeap BB, Hankey GJ, et al. 2008b. Low free testosterone concentration as a potentially treatable cause of depressive symptoms in older men. Arch Gen Psychiatry 65: 283289.

Amieva H, Le Goff M, Millet X, et al. 2008. Prodromal Alzheimer's disease: successive emergence of the clinical symptoms. Ann Neurol 64: 492-498.

Crum RM, Anthony JC, Bassett SS, et al. 1993. Population-based norms for the Mini-Mental State Examination by age and educational level. JAMA 269: 2386-2391.

Diniz BS, Butters MA, Albert SM, et al. 2013. Late-life depression and risk of vascular dementia and Alzheimer's disease: systematic review and meta-analysis of community-based cohort studies. Br J Psychiatry 202: 329-335.

Holman CD, Bass AJ, Rosman DL, et al. 2008. A decade of data linkage in Western Australia: strategic design, applications and benefits of the WA data linkage system. Aust Health Rev 32: 766-777.

Holman CD, Bass AJ, Rouse IL, et al. 1999. Population-based linkage of health records in Western Australia: development of a health services research linked database. Aust N Z J Public Health 23: 453-459.

Knopman DS, Roberts RO, Geda YE, et al. 2010. Validation of the telephone interview for cognitive status-modified in subjects with normal cognition, mild cognitive impairment, or dementia. Neuroepidemiology 34: 34-42.

Kohler S, Thomas AJ, Lloyd A, et al. 2010. White matter hyperintensities, cortisol levels, brain atrophy and continuing cognitive deficits in late-life depression. Br J Psychiatry 196: 143-149.

Korten NC, Penninx BW, Kok RM, et al. 2014. Heterogeneity of late-life depression: relationship with cognitive functioning. Int Psychogeriatr 26: 953-963.

Lautenschlager NT, Cox KL, Flicker L, et al. 2008. Effect of physical activity on cognitive function in older adults at risk for Alzheimer disease: a randomized trial. JAMA 300: 1027-1037. 
Li G, Wang LY, Shofer JB, et al. 2011. Temporal relationship between depression and dementia: findings from a large community-based 15-year follow-up study. Arch Gen Psychiatry 68: 970977.

Murray CJ, Vos T, Lozano R, et al. 2012. Disability-adjusted life years (DALYs) for 291 diseases and injuries in 21 regions, 1990-2010: a systematic analysis for the Global Burden of Disease Study 2010. Lancet 380: 2197-2223.

Norman PE, Flicker L, Almeida OP, et al. 2009. Cohort Profile: The Health In Men Study (HIMS). Int J Epidemiol 38: 48-52.

Norton S, Matthews FE, Barnes DE, et al. 2014. Potential for primary prevention of Alzheimer's disease: an analysis of population-based data. Lancet Neurol 13: 788-794.

Petersen RC, Caracciolo B, Brayne C, et al. 2014. Mild cognitive impairment: a concept in evolution. J Intern Med 275: 214-228.

Prina AM, Huisman M, Yeap BB, et al. 2013. Association between depression and hospital outcomes among older men. CMAJ 185: 117-123.

Saczynski JS, Beiser A, Seshadri S, et al. 2010. Depressive symptoms and risk of dementia: the Framingham Heart Study. Neurology 75: 35-41.

Sloane PD, Zimmerman S, Suchindran C, et al. 2002. The public health impact of Alzheimer's disease, 2000-2050: potential implication of treatment advances. Annu Rev Public Health 23: 213-231.

Snowdon DA, Greiner LH, Mortimer JA, et al. 1997. Brain infarction and the clinical expression of Alzheimer disease. The Nun Study. JAMA 277: 813-817.

Vilalta-Franch J, Lopez-Pousa S, Llinas-Regla J, et al. 2013. Depression subtypes and 5-year risk of dementia and Alzheimer disease in patients aged 70 years. Int J Geriatr Psychiatry 28: 341-350.

Zilkens RR, Spilsbury K, Bruce DG, et al. 2009. Linkage of hospital and death records increased identification of dementia cases and death rate estimates. Neuroepidemiology 32: 61-69. 
Table 1. Sociodemographic, lifestyle and clinical characteristics of older men according to the presence of clinically significant depressive symptoms.

\begin{tabular}{|c|c|c|c|c|c|}
\hline & \multicolumn{3}{|c|}{ Clinically significant depression } & \multirow{2}{*}{$\frac{\text { Statistic }}{\text { chi-square (df) }}$} & \multirow[t]{2}{*}{ p-value } \\
\hline & $\begin{array}{c}\text { No } \\
\mathrm{N}=3788 \\
\mathrm{n}(\%)\end{array}$ & $\begin{array}{c}\text { Past } \\
\mathrm{N}=527 \\
\mathrm{n}(\%)\end{array}$ & $\begin{array}{c}\text { Current } \\
\mathrm{N}=253 \\
\mathrm{n}(\%)\end{array}$ & & \\
\hline Age (in years): & $1430(37.7)$ & 187 (35.5) & $69(27.3)$ & $20.95(4)$ & $<0.001$ \\
\hline $75-79$ & $1623(42.8)$ & 229 (43.4) & 108 (42.7) & & \\
\hline$\geq 80$ & $735(19.4)$ & $111(21.1)$ & $76(30.0)$ & & \\
\hline Australian born & $1888(49.8)$ & $293(55.6)$ & $101(39.9)$ & $16.92(2)$ & $<0.001$ \\
\hline Education: $\geq$ High school & $1814(47.9)$ & $274(52.0)$ & $91(36.0)$ & $17.91(2)$ & $<0.001$ \\
\hline Smoking: & $1286(33.9)$ & $154(29.2)$ & 49 (19.4) & $29.28(4)$ & $<0.001$ \\
\hline Past & $2313(61.1)$ & 343 (65.1) & $182(71.9)$ & & \\
\hline Current & $189(5.0)$ & $30(5.7)$ & $22(8.7)$ & & \\
\hline Hypertension & $3106(82.0)$ & $422(80.1)$ & $205(81.0)$ & $1.23(2)$ & 0.541 \\
\hline Diabetes & $541(14.3)$ & $85(16.2)$ & $61(24.2)$ & $18.60(2)$ & $<0.001$ \\
\hline Coronary heart disease & $926(24.5)$ & $142(27.1)$ & $91(36.1)$ & $17.52(2)$ & $<0.001$ \\
\hline Stroke & $314(8.3)$ & $67(12.8)$ & $55(21.8)$ & $46.67(2)$ & $<0.001$ \\
\hline
\end{tabular}


Table 2. Cognitive outcome of participants according to their exposures five years earlier.

\begin{tabular}{|c|c|c|c|c|c|}
\hline & $\begin{array}{c}\text { No impairment } \\
\mathrm{N}=1126\end{array}$ & $\begin{array}{c}\text { MCI } \\
\mathrm{N}=642\end{array}$ & $\begin{array}{c}\text { Cognitive } \\
\text { Impairment } \\
\mathrm{N}=534 \\
\end{array}$ & $\begin{array}{l}\text { Dead } \\
\mathrm{N}=811\end{array}$ & $\begin{array}{l}\text { Unavailable } \\
\mathrm{N}=1455\end{array}$ \\
\hline & n (\%) & $\begin{array}{c}\mathrm{n}(\%) \\
\mathrm{RR}(95 \% \mathrm{CI})\end{array}$ & $\begin{array}{c}\mathrm{n}(\%) \\
\mathrm{RR}(95 \% \mathrm{CI})\end{array}$ & $\begin{array}{c}\mathrm{n}(\%) \\
\text { RR (95\%CI) }\end{array}$ & $\begin{array}{c}\mathrm{n}(\%) \\
\mathrm{RR}(95 \% \mathrm{CI}) \\
\end{array}$ \\
\hline Age (in years) & $\begin{array}{c}546(48.5) \\
1\end{array}$ & $\begin{array}{c}252(39.2) \\
1\end{array}$ & $\begin{array}{c}178(33.3) \\
1\end{array}$ & $\begin{array}{c}163(20.1) \\
1\end{array}$ & $\begin{array}{c}547(37.6) \\
1\end{array}$ \\
\hline $75-79$ & $\begin{array}{c}463(41.1) \\
1 \\
\end{array}$ & $\begin{array}{c}294(45.8) \\
1.38(\mathbf{1 . 1 2 - 1 . 7 0 )}\end{array}$ & $\begin{array}{c}252(47.2) \\
1.67(1.33-2.10) \\
\end{array}$ & $\begin{array}{c}330(40.7) \\
2.39(1.91-3.00) \\
\end{array}$ & $\begin{array}{c}621(42.7) \\
1.34(\mathbf{1 . 1 3 - 1 . 5 9 )} \\
\end{array}$ \\
\hline$\geq 80$ & $\begin{array}{c}117(10.4) \\
1 \\
\end{array}$ & $\begin{array}{c}96(14.9) \\
1.78(1.31-2.42)\end{array}$ & $\begin{array}{c}104(19.5) \\
2.73(1.99-3.73) \\
\end{array}$ & $\begin{array}{c}318(39.2) \\
\mathbf{9 . 1 0}(\mathbf{6 . 9 2 - 1 1 . 9 8 )}\end{array}$ & $\begin{array}{c}287(19.7) \\
2.45(1.92-3.13) \\
\end{array}$ \\
\hline Australian born & $\begin{array}{c}591(52.5) \\
1 \\
\end{array}$ & $\begin{array}{c}343(53.4) \\
1.04(0.86-1.26) \\
\end{array}$ & $\begin{array}{c}263(49.2) \\
0.88(0.71-1.08) \\
\end{array}$ & $\begin{array}{c}401(49.4) \\
0.89(0.74-1.06) \\
\end{array}$ & $\begin{array}{c}684(47.0) \\
\mathbf{0 . 8 0}(\mathbf{0 . 6 9 - 0 . 9 4 )} \\
\end{array}$ \\
\hline$\geq$ High school & $\begin{array}{c}694(61.7) \\
1 \\
\end{array}$ & $\begin{array}{c}323(50.3) \\
0.63(0.52-0.76) \\
\end{array}$ & $\begin{array}{c}203(38.0) \\
0.38(0.31-0.47) \\
\end{array}$ & $\begin{array}{c}339(41.8) \\
\mathbf{0 . 4 5}(0.37-0.54)\end{array}$ & $\begin{array}{c}620(42.6) \\
0.46(0.39-0.54) \\
\end{array}$ \\
\hline Smoking: & $\begin{array}{c}418(37.1) \\
1 \\
\end{array}$ & $\begin{array}{c}216(33.6) \\
1 \\
\end{array}$ & $\begin{array}{c}182(34.1) \\
1 \\
\end{array}$ & $\begin{array}{c}198(24.4) \\
1 \\
\end{array}$ & $\begin{array}{c}475(32.6) \\
1 \\
\end{array}$ \\
\hline Past & $\begin{array}{c}675(59.9) \\
1 \\
\end{array}$ & $\begin{array}{c}404(62.9) \\
1.16(0.94-1.42) \\
\end{array}$ & $\begin{array}{c}334(62.5) \\
1.14(0.91-1.41) \\
\end{array}$ & $\begin{array}{c}536(66.1) \\
1.68(1.37-2.05) \\
\end{array}$ & $\begin{array}{c}889(61.1) \\
1.09(0.92-1.29) \\
\end{array}$ \\
\hline Current & $\begin{array}{c}33(2.9) \\
1 \\
\end{array}$ & $\begin{array}{c}22(3.4) \\
1.29(0.73-2.27)\end{array}$ & $\begin{array}{c}18(3.4) \\
1.25(0.69-2.28)\end{array}$ & $\begin{array}{c}77(9.5) \\
4.93(3.17-7.66)\end{array}$ & $\begin{array}{c}91(6.2) \\
\mathbf{2 . 4 3 ( 1 . 6 0 - 3 . 6 9 )}\end{array}$ \\
\hline Hypertension & $\begin{array}{c}912(81.0) \\
1 \\
\end{array}$ & $\begin{array}{c}521(81.1) \\
1.01(0.79-1.29) \\
\end{array}$ & $\begin{array}{c}416(77.9) \\
0.83(0.64-1.07) \\
\end{array}$ & $\begin{array}{c}665(82.0) \\
1.07(0.85-1.35) \\
\end{array}$ & $\begin{array}{c}1219(83.8) \\
1.21(0.99-1.49) \\
\end{array}$ \\
\hline Diabetes & $\begin{array}{c}137(12.2) \\
1 \\
\end{array}$ & $\begin{array}{c}93(14.5) \\
1.22(0.92-1.62) \\
\end{array}$ & $\begin{array}{c}97(18.2) \\
\mathbf{1 . 6 0}(\mathbf{1 . 2 0 - 2 . 1 2}) \\
\end{array}$ & $\begin{array}{c}142(17.5) \\
1.53(1.19-1.98) \\
\end{array}$ & $\begin{array}{c}218(15.1) \\
\mathbf{1 . 2 8}(\mathbf{1 . 0 1 - 1 . 6 0 )} \\
\end{array}$ \\
\hline Coronary heart disease & $\begin{array}{c}250(22.3) \\
1\end{array}$ & $\begin{array}{c}137(21.4) \\
0.95(0.75-1.20)\end{array}$ & $\begin{array}{c}125(23.4) \\
1.07(0.84-1.37) \\
\end{array}$ & $\begin{array}{c}289(35.8) \\
1.94(1.59-2.38)\end{array}$ & $\begin{array}{c}358(24.7) \\
1.15(0.95-1.38) \\
\end{array}$ \\
\hline Stroke & $\begin{array}{c}78(6.9) \\
1\end{array}$ & $\begin{array}{c}47(7.3) \\
1.06(0.73-1.54)\end{array}$ & $\begin{array}{c}49(9.2) \\
1.36(0.93-1.97)\end{array}$ & $\begin{array}{c}110(13.6) \\
2.12(1.56-2.87)\end{array}$ & $\begin{array}{c}152(10.5) \\
1.57(\mathbf{1 . 1 8 - 2 . 0 9})\end{array}$ \\
\hline
\end{tabular}

RR: risk rate derived from a multinomial logistic regression. 95\%CI: 95\% confidence interval of the risk rate.

The bold print highlights statistically significant associations $(\mathrm{p}<0.05)$. 
Table 3. Risk rate of mild cognitive impairment (MCI), cognitive impairment, death and unavailability for assessment according to depression status of older men five years earlier.

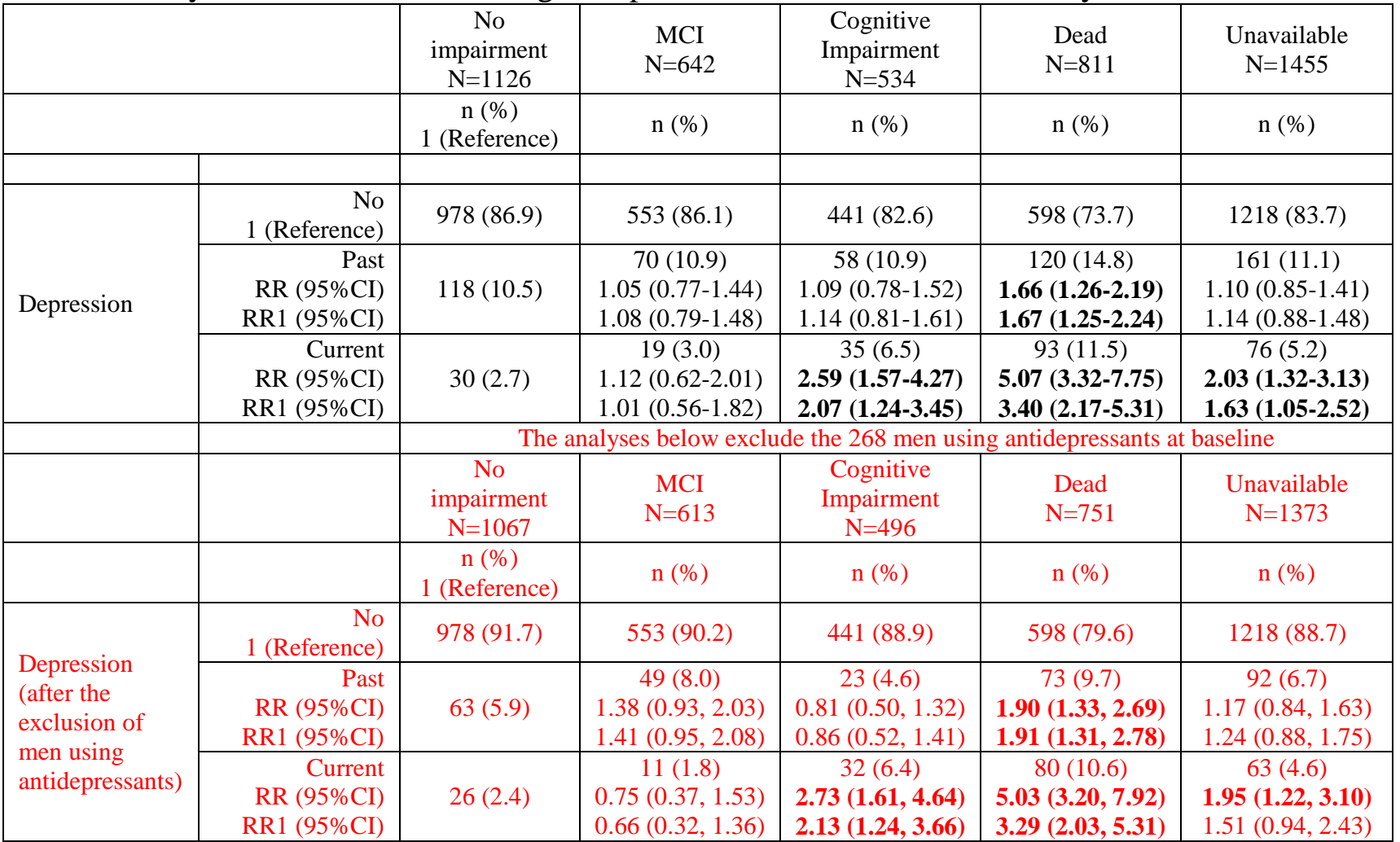

RR: Crude risk rate derived from a multinomial logistic regression. 95\%CI: 95\% confidence interval of the risk rate.

RR1: risk rate adjusted for age group, country of birth, education, smoking history, and prevalence hypertension, diabetes, coronary heart disease and stroke.

The bold print highlights statistically significant associations $(\mathrm{p}<0.05)$. 